LA W RENCE LIVERM ORE NATIONAL LABORATORY

MOSSFRAC: An anisotropic 3D fracture model

Willliam C. Moss, JoAnne L. Levatin

August 15, 2006 
This document was prepared as an account of work sponsored by an agency of the United States Government. Neither the United States Government nor the University of California nor any of their employees, makes any warranty, express or implied, or assumes any legal liability or responsibility for the accuracy, completeness, or usefulness of any information, apparatus, product, or process disclosed, or represents that its use would not infringe privately owned rights. Reference herein to any specific commercial product, process, or service by trade name, trademark, manufacturer, or otherwise, does not necessarily constitute or imply its endorsement, recommendation, or favoring by the United States Government or the University of California. The views and opinions of authors expressed herein do not necessarily state or reflect those of the United States Government or the University of California, and shall not be used for advertising or product endorsement purposes.

This work was performed under the auspices of the U.S. Department of Energy by University of California, Lawrence Livermore National Laboratory under Contract W-7405-Eng-48. 


\title{
MOSSFRAC: An anisotropic 3D fracture model
}

\author{
William C. Moss \\ JoAnne L. Levatin
}

August 9, 2006

Despite the intense effort for nearly half a century to construct detailed numerical models of plastic flow and plastic damage accumulation, models for describing fracture, an equally important damage mechanism still cannot describe basic fracture phenomena. Typical fracture models set the stress tensor to zero for tensile fracture and set the deviatoric stress tensor to zero for compressive fracture. One consequence is that the simple case of the tensile fracture of a cylinder under combined compressive radial and tensile axial loads is not modeled correctly. The experimental result is a cylinder that can support compressive radial loads, but no axial load, whereas, the typical numerical result is a cylinder with all stresses equal to zero. This incorrect modeling of fracture locally also has a global effect, because material that is fracturing produces stress release waves, which propagate from the fracture and influence the surrounding material. Consequently, it would be useful to have a model that can describe the stress relief and the resulting anisotropy due to fracture.

MOSSFRAC is a material model that simulates three-dimensional tensile and shear fracture in initially isotropic elastic-plastic materials, although its framework is also amenable to initially anisotropic materials. It differs from other models by accounting for the effects of cracks on the constitutive response of the material, so that the previously described experiment, as well as complicated fracture scenarios are simulated more accurately. The model is implemented currently in the LLNL hydrocodes DYNA3D, PARADYN, and ALE3D. The purpose of this technical note is to present a complete qualitative description of the model and quantitative descriptions of salient features. 


\section{Model Description}

The model allows both tensile and shear fracturing. Tensile fracture occurs when the maximum principal stress exceeds a user-defined threshold value. Shear fracture occurs when the equivalent plastic strain exceeds a user-defined value. We define an orthonormal set of three crack directions per computational zone. There can be only one crack in each direction, so that there are no more than three cracks per zone. Any combination of shear/tensile cracks is allowed. The crack opening direction is normal to the most tensile principal stress, even if that stress is compressive, so that closed shear cracks can be created. The orientation of the crack triad is determined as the cracks form. The initial orientation of the triad is arbitrary. The first time that the tensile or shear fracture criterion is reached, the most tensile principal stress direction becomes one of the crack triad directions. The remaining two are orthogonal to each other and coplanar in a plane normal to the first direction until a second crack forms, which defines the second direction of the orthonormal triad. The triad is determined completely from the cross product of the first two directions. The crack algebra is formulated so that the cracks open and close independently, while maintaining zero traction on open cracks. Strength is maintained in the uncracked directions. The difference between the material response to applied deformations in the cracked and uncracked directions generates anisotropy.

The model has a general structure that is typical of many material models in that an elastic strain increment results in a stress increment. By partitioning the total applied strain increment $d \epsilon$ into elastic $(e)$, plastic $(p)$, and crack $(c)$ strain increments, we isolate the elastic strain increment. We assume a linear relationship between these tensor increments and write

$$
d \epsilon^{e}=d \epsilon-d \epsilon^{p}-d \epsilon^{c} .
$$

It is convenient (and traditional) to further partition the elastic strain increment into deviatoric and dilatational components, which allows the deviatoric stress increment to be calculated using the plasticity model, and the total dilatational stress, or pressure, to be calculated using the equation of state. We write

$$
\begin{aligned}
d S & =2 G d e^{\prime} \text { and } \\
P & =f\left(V^{e}, E\right),
\end{aligned}
$$

where $e^{\prime}$ is the deviatoric elastic strain, $S$ is the deviatoric stress, $G$ is the current shear modulus, and the pressure $P$ is obtained from the equation of state $f$, which is a function of the elastic volume $V^{e}$ (total volume minus crack volume) and the internal energy $E$. The stress increment $d \sigma$ can be calculated from the elastic strain increment, when the values of $d \epsilon^{p}$ and $d \epsilon^{c}$ in Eq. (1) are determined, because 
the total applied strain increment $d \epsilon$ is known. When the crack strains are zero, the plastic strains can be determined easily and directly by various methods, such as the radial return method. However, for the general case there is no analytic method to determine plastic strains and crack strains that produce a stress state that simultaneously satisfies plastic yield and traction-free criteria.

Figure 1 shows the split operator method we use for the combined effects of plasticity and fracture. The applied strain increment is processed sequentially, as shown in the figure. Part of the rationale for splitting the operators, as shown in the figure, is that the model was constructed originally for use in DYNA3D, which calculates plasticity first, then pressure.

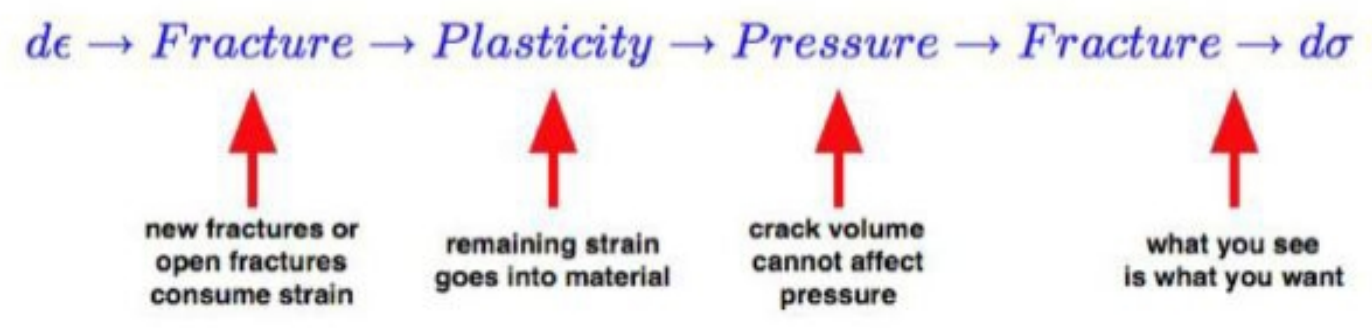

Figure 1: Split operator method for obtaining the stress increment from the strain increment. Plastic and crack strains cannot be determined simultaneously, so an approximate method must be used.

\section{- Fracture}

- uncracked material: A trial stress increment is calculated from the total applied strain increment, assuming isotropic elasticity and using the current elastic moduli (two elastic moduli: bulk and shear). A trial stress is obtained from the sum of the initial stress and the trial stress increment. This stress is rotated into the principal frame. If the stress exceeds the shear/tensile fracture criteria in one or more directions, then one or more cracks are created and the stress is relaxed, so that there are traction-free crack surfaces on open cracks.

- cracked material: The strain increment is rotated into the crack frame and is "consumed" by open cracks, i.e., open cracks either continue opening or consume enough strain to close. The remaining strain increment $d \epsilon-d \epsilon^{c}$ is used to compute a trial stress increment, using the current elastic moduli and assuming isotropic elasticity. A trial stress is obtained from the sum of the initial stress (rotated into the crack frame) and the trial stress increment. This stress is examined to see if 
it exceeds the shear/tensile fracture criteria, in any remaining unfractured directions. If the stress exceeds the shear/tensile fracture criteria in any of the unfractured directions, then cracks are created and the stress is relaxed to accommodate traction-free crack surfaces on open cracks.

The additional crack strain increment due to the stress relaxation is calculated using the stress increment that is required to zero the surface tractions, isotropic linear elasticity, and the current bulk and shear moduli. We allow either zero or infinite friction on closed cracks. The remaining strain increment $d \epsilon-d \epsilon^{c}$, where $d \epsilon^{c}$ is now the sum of all previously calculated crack strain increments, causes material strain and is used to calculate elasticplastic deformation by the Plasticity subroutine.

\section{- Plasticity}

The strain increment $d \epsilon-d \epsilon^{c}$ that is calculated by the Fracture subroutine is used to generate a stress increment and a trial stress. Consequently, any plasticity model can be used. We use the well known Steinberg-Guinan model, which consists of a pressure, density, and temperature dependent shear modulus, and a pressure, temperature, density, and plastic strain dependent yield strength. Both elastic and plastic responses are isotropic. Placing the stress deviators on the yield surface alters the total stress, which may no longer satisfy the traction-free condition on open cracks. Another pass through the fracture subroutine is required to reinforce the traction-free condition, but first, the pressure is updated.

\section{- Pressure}

The pressure is typically a tabular or an analytic function of the specific internal energy $E$ and the relative volume $\eta=V / V_{o}$, where $V$ is the volume and $V_{o}$ is a reference volume. If we consider the cylinder discussed earlier, then it is clear that the pressure should be a function of the elastic or material deformation $\eta^{e}$, because changes in the crack volume should not affect the pressure. We write $\eta^{e}=\left(V-V^{c}\right) / V_{o}$, where $V^{c}$ is the crack volume, which can be computed from the three crack strains $\epsilon_{i}^{c}$. We write

$$
V^{c}=V_{o}\left(\exp \left(\epsilon_{1}^{c}+\epsilon_{2}^{c}+\epsilon_{3}^{c}\right)-1\right)
$$

A complete discussion of the crack strain formulation is given in the Appendix. 


\section{- Fracture}

The modifications of the stress by the Plasticity and Pressure subroutines can result in a stress state that $(i)$ no longer satisfies the traction-free conditions for open cracks, $(i i)$ creates new cracks, and (iii) closes open cracks. Consequently, another call to the Fracture subroutine using an applied strain increment equal to zero re-equilibrates the stresses. This final pass through the Fracture subroutine is done primarily for aesthetic reasons, so that the visualization of zones with open cracks is consistent.

This second pass through the Fracture subroutine results in a final stress state.

\section{Symmetry Breaking (representation of inhomogeneity)}

An axisymmetric simulation produces an axisymmetric result. An axisymmetric experiment does not produce axisymmetric data. Symmetry breaking occurs, because at some length scale, either the experiment is not truly axisymmetric and/or the material is not homogeneous. We model symmetry breaking by allowing the tensile and/or shear fracture criteria to differ from zone to zone. At initialization, each zone is assigned a random number $x \in[0,1]$. A multiplier $W$ is calculated for each zone using a one-parameter Weibull distribution. We write

$$
W=\left(\frac{m \ln (1-x)}{1-m}\right)^{1 / m}
$$

where the most probable value of the distribution equals one and $m$ controls the width of the distribution $(m>1$ : when $m=10, \mathrm{FWHM}=0.25$; when $m=30$, FWHM $=0.07)$. The material tensile strength and/or equivalent plastic strain to initiate shear fracture are multiplied by the zonal value of $W$ to create global inhomogeneity.

\section{Appendix: Crack Strains}

In this section we discuss the equations that are used to calculate the crack strains. Although the full model has equations for the complete crack strain tensor (with either no friction or infinite friction on closed cracks), for simplicity, we discuss only crack opening and closing, for 1, 2, and 3 cracks. The conditions for determining which cracks are opening/closing are also not discussed here, but are contained in the complete model. 


\section{- Definition}

The applied strain increment either opens/closes cracks and/or deforms material, so the definition of the crack strain must be consistent with the definition of material strain. This means that total strains are the sums of the increments, and allows the applied strain increment to be partitioned arbitrarily. The difficulty with this formulation occurs when we want to compute actual crack openings and volumes from the strains. We proceed as follows. We use "true" strains, so that an element of current length $l$ that is extended by an amount $d l$ is strained an amount $d l / l$. (This strain definition is consistent with that used typically in hydro codes to describe material strain increments.) Again, using this one-dimensional deformation for simplicity, we write the crack strain as $d \epsilon^{c}=d l^{c} / l$, where $d l^{c}$ is the incremental crack opening and $l$, which is the current length of the zone, equals the sum of the original length $l_{o}$, the elastic stretch $l^{e l}$, and the crack opening $l^{c}$. We write $l=l_{o}+l^{e l}+l^{c}$. The elastic stretch should be small when there are open cracks, which yields an approximate but analytic expression of the crack strain, as a function of the crack opening. We write

$$
\epsilon^{c}=\int_{0}^{l^{c}} \frac{d l^{\prime}}{l} \approx \int_{0}^{l^{c}} \frac{d l^{\prime}}{l_{o}+l^{\prime}}=\ln \frac{l_{o}+l^{c}}{l_{o}} .
$$

Eq.(3) follows directly from Eq.(5). The generalized equations for one, two, and three open cracks are

- One crack open

$$
d \epsilon_{1}^{c}=\delta \epsilon_{1}^{c}+\frac{\tilde{\sigma}_{1}}{\lambda+2 G}
$$

\section{- Two cracks open}

$$
\begin{aligned}
& d \epsilon_{1}^{c}=\delta \epsilon_{1}^{c}+\frac{(\lambda+2 G) \tilde{\sigma}_{1}-\lambda \tilde{\sigma}_{2}}{4 G(G+\lambda)} \text { and } \\
& d \epsilon_{2}^{c}=\delta \epsilon_{2}^{c}+\frac{(\lambda+2 G) \tilde{\sigma}_{2}-\lambda \tilde{\sigma}_{1}}{4 G(G+\lambda)}
\end{aligned}
$$

\section{- Three cracks open}

$$
\begin{aligned}
& d \epsilon_{1}^{c}=\delta \epsilon_{1}^{c}+\frac{(\lambda+2 G) \tilde{\sigma}_{1}+\lambda\left(\tilde{\sigma}_{1}-\tilde{\sigma}_{2}-\tilde{\sigma}_{3}\right)}{6 K G}, \\
& d \epsilon_{2}^{c}=\delta \epsilon_{2}^{c}+\frac{(\lambda+2 G) \tilde{\sigma}_{2}+\lambda\left(\tilde{\sigma}_{2}-\tilde{\sigma}_{1}-\tilde{\sigma}_{3}\right)}{6 K G}, \quad \text { and } \\
& d \epsilon_{3}^{c}=\delta \epsilon_{3}^{c}+\frac{(\lambda+2 G) \tilde{\sigma}_{3}+\lambda\left(\tilde{\sigma}_{3}-\tilde{\sigma}_{1}-\tilde{\sigma}_{2}\right)}{6 K G},
\end{aligned}
$$


where $G$ and $K$ are the current shear and bulk moduli, $\lambda=K-2 G / 3, \delta \epsilon_{i=1,2,3}^{c}$ is the portion (if any) of the applied strain increment (rotated into the crack frame) that has been consumed previously by crack opening/closing, and $\tilde{\sigma}_{i=1,2,3}$ is the current trial stress. 